\title{
Food Hygiene Rating Scheme (FHRS) Food and You 2: Wave 2
}

\author{
Area of research interest: Food and You 2 \\ Project status: Completed \\ Authors: Dr Beth Armstrong, Lucy King, Ayla Ibrahimi, Robin Clifford, Mark Jitlal \\ Date published: 16 December 2021 \\ DOI: https://doi.org/10.46756/sci.fsa.ozf866
}

\section{Background}

The Food Hygiene Rating Scheme (FHRS) is run in partnership between the Food Standards Agency and Local Authorities and provides information on the standards of hygiene found in food businesses at the time they are inspected. The scheme covers businesses providing food directly to consumers, such as restaurants, pubs, cafés, takeaways, hotels, hospitals, schools and other places people eat away from home, as well as supermarkets and other food shops. In Wales, the scheme also includes businesses that trade only with other businesses, for example, manufacturers.

Food and You 2: Wave 2 is the first wave of data collection to include questions relating to the FHRS. The Food and You 2 survey has replaced the biennial Food and You survey (2010-2018), biannual Public Attitudes Tracker (2010-2019) and the Food Hygiene Rating Scheme (FHRS) Consumer Attitudes Tracker (2014-2019). We previously commissioned the FHRS Consumer Attitudes Tracker survey to monitor consumer awareness, attitudes towards and use of the scheme. The survey moved from a biannual basis to an annual basis from 2017 onwards. Due to differences in the question content, presentation and mode of response, direct comparisons should not be made between these earlier surveys and Food and You 2.

\section{Results}

\section{Awareness and recognition of the scheme}

- $87 \%$ of respondents reported that they had heard of the Food Hygiene Rating Scheme

- Of those who had heard of the FHRS, the most common place respondents had come across the FHRS was a food hygiene rating sticker displayed at a food business (83\%)

- Of those who had heard of the FHRS, over a third (36\%) of respondents had come across the FHRS on a food business' website

- When shown an image of the food hygiene rating sticker, $90 \%$ of respondents reported that they had seen the sticker before

\section{Understanding the use of FHRS}

- Of those who had heard of the FHRS, approximately half (51\%) of respondents had checked the food hygiene rating of a food business in the previous 12 months (either at the business premises or online)

- Of those who have checked the food hygiene rating of a food business, most had checked the food hygiene rating of takeaways (70\%) and restaurants (64\%) 
- Of those who have checked the food hygiene rating of a food business, most had checked the rating by looking at the food hygiene rating sticker displayed at the food business $(78 \%)$

\section{Use of the FHRS in decision making}

- Of those who had heard of the FHRS, most respondents said they would still eat at a restaurant or takeaway if they saw a food hygiene rating sticker with a rating of 4 (good) $(95 \%)$ or 3 (generally satisfactory) (63\%)

- Respondents were asked what they would usually consider the lowest acceptable food hygiene rating when considering buying food from somewhere. $41 \%$ of respondents would consider a rating of 4 (good) as the lowest acceptable rating and $43 \%$ of respondents would consider 3 (generally satisfactory)

- Of those who had heard of the FHRS, $61 \%$ of respondents would be less likely to eat at a food business that did not have the food hygiene rating sticker present at the entrance

\section{Views on mandatory display}

- Of the respondents who had heard of the FHRS, 95\% thought that food businesses should be required by law to display their food hygiene rating at their premises

- Of the respondents who had heard of the FHRS, 95\% thought that businesses providing an online food ordering service should display their food hygiene rating where it can clearly be seen by customers before they order food

Research report

\section{England, Northern Ireland and Wales}

PDF

View FHRS Food and You 2 Wave 2 as PDF(Open in a new window) (650.46 KB) 